\title{
Análisis subléxico del verbo de movimiento "Pasear"1
}

Por: Mag. Gabriel Valdés-León², Universidad Católica Silva Henríquez, Chile

Recibido: $\quad 22$ de enero, 2019.

Aceptado: $\quad 30$ de abril, 2019.

\section{Resumen}

En este trabajo se presenta un estudio cuyo objetivo es proponer una definición mínima del verbo "pasear" como verbo de movimiento, amediante el análisis combinatorio con sustantivos de compleja delimitación, a saber, montaña-monte y mar-océano. Para ello se propone, en primer lugar, un marco teórico que considera las ideas principales de la Teoría del Lexicón Generativo (TLG) y una revisión de los estudios más relevantes respecto del verbo "pasear" como verbo de movimiento, para dar paso a un análisis combinatorio realizado a partir de las apariciones en el CORPES XXI. Posteriormente, se relacionan estos resultados, de índole lexicológica, con eventuales aplicaciones lexicográficas, en algunos casos de difícil delimitación. Dentro de los principales resultados, este trabajo propone una definición del verbo "pasear" que considera la inclusión del rasgo $[ \pm$ recreativo] como parte de la estructura de qualia, lo que podría contribuir no solo con el establecimiento de límites en entradas que poseen difusas líneas fronterizas, sino también en los estudios de semántica léxica y composicional.

\section{A sub-lexical analysis of the movement verb "pasear"}

\section{Abstract}

This paper presents a study that aimed to propose a minimal definition of the verb "pasear" as a verb of movement through the combinatorial analysis with nouns of complex delimitation, namely as montaña-monte and mar-océano. For this, we propose, first of all, a theoretical framework that considers the main ideas of the Generative Lexicon Theory (GLT) and a review of the most relevant studies regarding the verb "pasear" as a movement verb, to make way for an analysis combinatorial made from the appearances in the CORPES XXI. Later, these results are related, of lexicological nature, with possible lexicographical applications in some cases of difficult delimitation. Within the main results, this work proposes a definition of the verb "pasear" that considers the inclusion of the $[ \pm$ recreational] feature as part of the qualia structure, which could contribute not only with the establishment of limits in entries that have diffuse border lines, but also in the studies of lexical and compositional semantics.

1 Este trabajo es una síntesis del Trabajo Final del Máster en Lexicografía Hispánica, realizado por el autor e impartido en conjunto por la RAE, ASALE y la Universidad de León.

2 Gabriel Valdés-León es Magíster en Lingüística y Máster en Lexicografía Hispánica, graduado en la Universidad de León, España. Actualmente realiza su doctorado en Estudios Lingüísticos y Literarios en sus Contextos Socioculturales, en la Universidad de Las Palmas de Gran Canaria, España. Labora como académico en la Universidad Católica Silva Henríquez, Chile. Contacto: g_valdes@icloud.com.
Gabriel Valdés-León. Análisis subléxico del verbo de movimiento "pasear". Revista Comunicación. Año 40, volumen 28, número 1, enero-junio, 2019. Instituto Tecnológico de Costa Rica. ISSN: 0379-3974, / e-ISSN1659-3820.
PALABRAS CLAVE:

semántica, terminología, vocabulario, lexicografía, investigación lingüística, teoría lingüística.

\section{KEY WORDS:}

semantics, terminology, vocabulary, lexicography, linguistic research, linguistic theory. 


\section{INTRODUCCIÓN}

Al revisar algunas entradas del Diccionario de la Lengua Española (DLE), se puede observar una serie de términos que carecen de la exhaustividad necesaria en el momento de establecer límites semánticos, sobre todo en aquellos casos en que estas palabras parecieran formar parte de un continuum de difícil delimitación: ¿dónde está la diferencia entre el referente de mar y el de océano? ¿En su extensión? ¿En su cercanía con la costa? El diccionario académico ofrece como primera acepción de mar, la siguiente: "masa de agua salada que cubre la mayor parte de la superficie terrestre". En tanto, para océano propone "grande y dilatado mar que cubre la mayor parte de la superficie terrestre". Evidentemente, estas acepciones no ayudan demasiado al usuario del diccionario que quiere saber dónde se baña cada vez que va a la playa.

Una situación similar ocurre con monte y montaña. Si bien el hablante podría verse tentado a recurrir a manuales especializados que zanjen la discusión estableciendo la diferencia en una altura determina$\mathrm{da}^{3}$, es opinión de quien escribe que un diccionario general debe nutrir sus definiciones a partir del uso que se evidencia en la realidad lingüística, pues, al final, resultan ser los hablantes quienes identifican rasgos particulares en un accidente geográfico que nos permiten denominarlo de una u otra manera.

Al observar las entradas monte y montaña en el DLE, lo primero en lo que podemos reparar es en su parentesco etimológico: mientras que monte proviene del latín mons, montis, montaña lo hace del latín vulgar *montanea que, a su vez, proviene de mons, montis. No parece extraño, entonces, que la primera acepción sea -salvo su género- exactamente igual en ambos, a saber, "gran elevación natural de terreno". Cabe, pues, preguntarse por qué no remite una entrada a la otra (si pueden ser considerados como sinónimos) o, si no lo son, qué diferencias se presentan entre ambos lemas, ya que no se establecen límites claros entre ellos.

Para abordar el problema de la falta de discriminación en las definiciones de los pares de palabras montaña-monte y mar-océano, se ha acudido al
Corpes XXI con el fin de identificar combinaciones sintácticas que puedan revelar rasgos mínimos presentes en las entradas. En esta búsqueda, el verbo "pasear" resultó particularmente decisivo en el momento de proponer un criterio que ofrezca una solución con sustento en la semántica, particularmente, desde la perspectiva de la Teoría del Lexicón Generativo (TLG). Gracias a este hallazgo, se propuso entregar una definición del verbo de movimiento "pasear" utilizando el análisis combinatorio con los sustantivos antes mencionados.

Sobre esta base, nuestro marco teórico ofrecerá, en primer lugar, algunos de los aspectos más relevantes de la TLG que nos permitirán abordar el problema anteriormente mencionado, a saber, la delimitación de las entradas montaña-monte y mar-océano (cf. infra §1.2). Luego, considerando la importancia del verbo "pasear" en el establecimiento de dichos límites, se repasará el tratamiento que este verbo ha recibido desde diversas áreas de la lingüística, las cuales se han dedicado a estudiar los verbos de movimiento.

Así, luego de establecer los principales sustentos teóricos de este trabajo, se ofrecerá un breve acápite sobre la metodología utilizada para, posteriormente, exponer el proceso analítico y los resultados obtenidos.

\section{ANTECEDENTES TEÓRICOS}

Como se señaló anteriormente, en este trabajo se pretende estudiar el verbo "pasear" $y$, gracias a ello, ofrecer posibles implicaciones lexicográficas y lexicológicas que puedan surgir mediante el análisis combinatorio con sustantivos difíciles de delimitar, tales como montaña-monte y mar-océano. A partir de lo anterior, resulta necesario ofrecer algunas ideas clave sobre la TLG, teoría en la que se sustenta el presente estudio, pero no sin antes señalar cómo se relaciona con la perspectiva semántica estructuralista.

3 Por ejemplo, una elevación de terreno adquiere categoría de "montaña" desde los 1000 metros en España, mientras que en Alemania, Francia e Italia se exigen 700 metros (Delgado, García y Hontañón, 2010). 


\section{Perspectiva semántica-estructuralista}

Desde la semántica estructuralista, se propone la posibilidad de entender los significados de las palabras como un elemento divisible capaz de ser descompuesto en una serie de rasgos menores a través del análisis componencial. En este sentido, este tipo de análisis ofrece un modelo que, a través de relaciones de hiponimia/hiperonimia, entrega una representación esquemática que favorece la clasificación de elementos que pertenecen a un mismo campo semántico.

Específicamente desde el análisis componencial, los verbos de movimiento se pueden descompo- ner en semas (significados elementales) y sememas (conjunto de semas). Desde esta perspectiva, Meya (1976, p. 148) plantea que "los verbos de movimiento: subir, andar, pasear..., etc., son sememas que tienen en común varios semas, hecho que fundamenta su pertinencia a un mismo campo".

Esta misma autora propone una clasificación de los verbos de movimiento estudiados (entre ellos, "moverse", "ir", "pasear", "andar", "caminar", "correr"...) considerando semas genéricos y específicos, clasificación que se presenta en el siguiente esquema (Figura 1):

\section{Figura 1}

\section{Semas nucleares, genéricos y específicos de los verbos de movimiento estudiados}

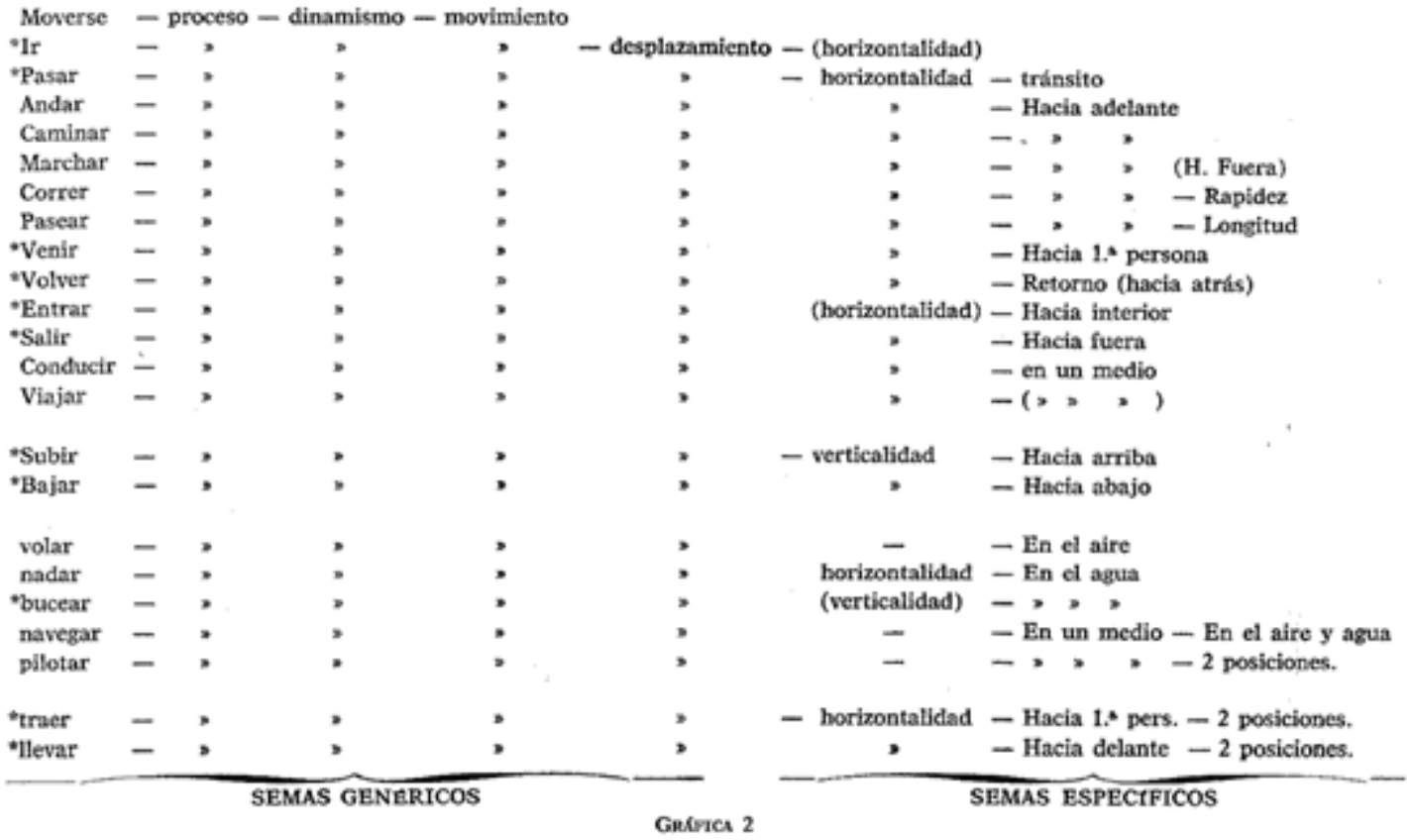

Fuente: Meya (1976, p. 148)

Si bien esta perspectiva posee una fuerte tradición en el desarrollo de la lingüística, hay que señalar que el análisis componencial que propone la lexemática evidenció una serie de dificultades (Ullman, 1962, citado en De Miguel, 2015): a) la inabarcabilidad de los rasgos necesarios para dar cuenta de la totalidad de los campos léxicos existentes; b) la dificultad de proponer rasgos distintivos en algunos campos léxicos, por ejemplo, el de los sentimientos; c) la complejidad de expresar las relaciones de significado en términos únicamente binarios; y d) la clasificación no prototípica de miembros de un campo léxico a una clase a la que pueden no pertenecer en el mundo real.

Ahora bien, una de las principales herencias de la perspectiva semántica estructural en los estudios 
lexicológicos actuales, es la defensa de un estudio de la lengua centrado en las palabras y no en los objetos y eventos del mundo. Al respecto, De Miguel (2015) señala:

Los modelos de inspiración estructural (incluidos los funcionales y generativistas) defienden un estudio del significado léxico centrado en definir las palabras y no los objetos o eventos del mundo a los que se refieren, punto básico de discrepancia con los semantistas cognitivos, para quienes la lengua, como una manifestación más de la cognición, incorpora el mundo en sus conceptualizaciones.

\section{La Teoría del Lexicón Generativo}

Indudablemente, uno de los mayores desafíos a los que se ve enfrentado el lexicógrafo implica buscar el equilibrio entre exhaustividad, por una parte, y claridad y concisión, por otra. Esta tarea resulta bastante complicada cuando se considera que uno de los principios elementales de la semántica es que las palabras adquieren significado "en contexto" (Martí, 2001). Debido a esto, una entrada alusiva al verbo "correr" puede incorporar un vastísimo número de acepciones según posibilidades combinatorias se encuentren: para comprobarlo, basta con mirar las más de 45 acepciones que recoge el DLE (2014).

Ante este panorama, han surgido propuestas desde la lexicología apoyadas, principalmente, en la TLG (Pustejovsky, 1995) con el objetivo de ofrecer una definición lexicográfica que considere los rasgos mínimos que hacen posible la totalidad de las combinaciones $^{4}$. Efectivamente, Pustejovsky plantea que el lexicón mental de los hablantes no está constituido por una extensa lista de significados, sino por un lexicón generativo que entrega principios y mecanismos generales con los cuales es posible actualizar el significado de una palabra en relación con el contexto en el que se presenta. En este sentido, "el lexicón no constituye un almacén estático de los sentidos de las palabras sino un com- ponente dinámico, flexible y sensible al contexto" (De Miguel, 2009).

La TLG supone cuatro niveles que constituyen un signo léxico: estructura argumental, eventiva, de tipificación léxica y de qualia. El primer nivel especifica el número de argumentos de un predicado, su realización sintáctica y su clase semántica; la estructura eventiva por su parte, indica el tipo de evento que denota un predicado; la de tipificación léxica, explica cómo una estructura léxica se relaciona con otras en el lexicón mental; y, por último, la estructura de qualia ofrece información sobre cómo está constituido el objeto o evento (quale constitutivo), cuáles son sus características diferenciadoras (quale formal), qué función o propósito posee (quale télico) y qué factores han estado implicados en su origen (quale agentivo) (De Miguel, 2009; Climent, 1999).

\section{METOdOLOGÍA}

Para llevar a cabo esta revisión se han seleccionado, en primer término, los lemas mar-océano y monte-montaña puesto que la difusa delimitación que ofrece el DLE nos sirve como punto de partida para llegar a definir el verbo "pasear". Ahora bien, entendiendo que el significado se presenta como un fenómeno contextual, se llevó a cabo una búsqueda en Corpes XXI para observar el comportamiento de estos sustantivos con dos verbos de movimiento: "llegar" y "pasear". Posteriormente, la baja frecuencia de coapariciones entre los lemas océano y "pasear", por una parte, y montaña y "pasear", por otra, provocó la indagación en combinaciones con los adjetivos alto, nevado, rocoso, inaccesible, salvaje e inhóspito para el primer par de palabras, y lejano, profundo, oscuro y amenazante para el segundo, con el fin de establecer un criterio de diferenciación.

Es importante señalar que, para efectos de esta investigación, se entenderá el término "coapariciones" como la relación de proximidad que existe entre dos lemas en un intervalo determinado, ya sea a la derecha o izquierda, en el decurso de un enunciado. Esto permite observar

$4 \quad$ Un claro ejemplo es el Diccionario Electrónico Multilingüe (Dicemto) creado por el grupo investigador UPSTAIRS, perteneciente a la Universidad Autónoma de Madrid. Puede consultarlo en el enlace https://www.uam.es/gruposinv/upstairs/diccionario.html. 
fenómenos contextuales que pueden llegar a ser relevantes, por ejemplo, la capacidad que posee el verbo "pasear" para condicionar la selección del resto de palabras que conforman el enunciado. A modo de ejemplo, es posible anticipar que el verbo "pasear" exige complementos conformados por realidades paseables y recreativas, vale decir, que compartan algunas características que les permitan relacionarse semánticamente. Cuando esto no ocurre, debe existir algún mecanismo que le dé sentido a la frase. Si bien se profundizará mucho más sobre esto en el apartado §5, es viable proponer un par de ejemplos tomados del Corpes XXI (la negrita es nuestra) (Figura 2):

Figura 2

\section{Ejemplos tomados de Corpes XXI}

- La Ley 16.088 fue el fruto de mi decisión de terminar con la peligrosísima extravagancia de pasear leonas y hasta una pantera.

- Lo que Buxton encuentra es algo inaudito: un león paseando por un camino.

Fuente: Corpus Corpes XXI (Corpes XXI, s.f.)

Los ejemplos anteriores han sido presentados con el objetivo de evidenciar la importancia de la metodología que aquí se ha seguido. En efecto, el criterio utilizado, más que identificar relaciones restrictivas de determinación (en el sentido tradicional de "coaparición"), permite identificar la relación entre los lemas que conforman un mismo enunciado y cómo, en contexto, se relacionan.

Sobre la base de lo anterior, vale destacar que, para llevar a cabo las combinaciones en Corpes XXI, se ha establecido un intervalo de proximidad de tres palabras a la derecha o izquierda en el momento de relacionar el verbo "pasear" con montaña-monte y cinco con mar-montaña. Esta diferencia responde a que la relación entre "pasear" y mar-océano es más bien indirecta: como se verá en los ejemplos, se puede pasear por el océano en un navío (no directamente), o bien, por su orilla. Debido a lo anterior, resulta necesario ampliar los criterios de proximidad y obtener mayor información contextual para aquellos casos en los que "pasear" y mar-oceáno forman parte de un mismo enunciado.
Finalmente, es menester señalar que aquí se ha adoptado una metodología inductiva, pues el punto de partida resulta ser las manifestaciones concretas del uso de la lengua, las que se han estudiado contextualmente para llegar a establecer patrones de comportamiento que permiten proponer aplicaciones lexicográficas.

\section{ANÁLISIS}

\section{1. montaña y monte con verbos de movimiento}

Al buscar monte en Corpes XXI, se encuentran 6 956 casos documentados de este lema. Si se combina con llegar, se presentan 64 casos, lo que equivale a menos del $1 \%$ del total de resultados. Si bien parece una cifra despreciable, a ningún hablante del español le resultará extraña una construcción como "Hasta que llegaron a un monte y vieron una casa...". Así, monte se combina perfectamente con un verbo de movimiento cuya estructura argumental es "N1 (llegar) a/hasta N2", cuyo quale formal de N1 es una entidad dinámica ("ellos", en el ejemplo) y una locación en el caso de N2 ("el monte") (UPSTAIRS, s.f.).

Por su parte, la combinación entre "pasear" y monte resulta aún menos productiva. En efecto, bajo las condiciones que se han establecido aquí, se despliegan siete casos combinatorios. Ahora bien, en un ejemplo como "su nueva vida se resume en tocar la guitarra (...), pasear por el monte con sus perros...", nuevamente se tiene un caso que, pese a las bajas apariciones, no representa un desafío interpretativo para un hablante del español.

Por otro lado, en el caso de montaña, se obtienen 13 312 casos documentados en Corpes XXI. Al combinar este lema con llegar, se consiguen 78 casos documentados y, tal como sucedió con monte, no evidencian ningún inconveniente interpretativo ni error gramatical: "Los tres llegarán a la montaña en 59 segundos de margen". Al combinar esta entrada con "pasear" se obtienen, sin embargo, resultados mucho más significativos para contribuir con la diferenciación entre montaña y monte. En efecto, la búsqueda arroja tan solo dos casos documentados, los que se exponen a continuación (Figura 3): 
Figura 3

Coapariciones "pasear" y montaña

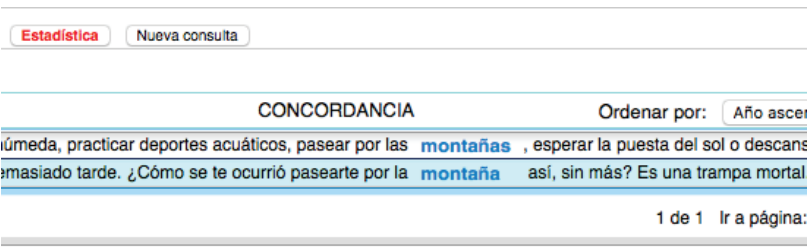

Fuente: Corpus Corpes XXI (Corpes XXI, s.f.)

De los dos casos entregados por Corpes XXI, hay una idea que se desprende del segundo ${ }^{5}$; por las montañas no se pasea!

\section{2. mar y océano con verbos de movimiento}

El comportamiento de los verbos "pasear" y "Ilegar" con los sustantivos mar y océano es bastante similar.
En efecto, el sustantivo mar presenta 31334 casos en 9803 documentos, una cantidad de apariciones muy alta en el Corpes XXI. Luego, si se relaciona el verbo "legar" con este mismo sustantivo, se obtienen 314 apariciones, una considerable cantidad si se compara con los 64 casos entre "llegar"-monte con los mismos criterios. Bastante menor es la frecuencia de aparición de entre "pasear" y mar, con tan solo 16 casos en 16 documentos.

Por su parte, el sustantivo océano presenta casi 5000 apariciones en el corpus de la Academia, cifra que disminuye a 51 casos cuando se combina este sustantivo con "llegar". Ahora bien, el dato realmente significativo -y que parece demostrar el comportamiento del verbo "pasear" con un grupo de sustantivos que presentan rasgos comunes- es el bajísimo número de coapariciones entre "pasear"océano: tan solo tres (Figura 4):

Figura 4

\section{Coapariciones "pasear" y océano}

\begin{tabular}{|c|c|c|}
\hline CONCORDANCIA & Ordenar por: & Año ascendente \\
\hline uro en la noche, y me imaginé a los señores del océano & \multicolumn{2}{|c|}{ paseando en la negrura como leones en la pradera africana. } \\
\hline o en el camarote, pero un golpe de mar la tiró al océano & \multicolumn{2}{|c|}{ mientras paseaba por cubierta y se ahogó irremisiblemente. I } \\
\hline , ya que la lluvia imposibilitaba pasearse junto al océano & \multicolumn{2}{|c|}{ o seguir un sendero en los jardines del Palacio de Cristal, que } \\
\hline
\end{tabular}

Fuente: Corpus Corpes XXI (Corpes XXI, s.f.)

Si se observan con detención los datos que ofrece Corpes XXI, se nota que, de los casos presentados, es posible descartar fácilmente el segundo, pues implica que alguien pasea por la cubierta de un barco y este, a su vez, navega por el océano. Por su parte, el tercero hace alusión a "pasearse junto al océano" vale decir, por la playa, aspecto que resulta muy interesante porque los datos del Corpes XXI señalan que los hablantes suelen pasear por "la orilla/borde del mar" mucho más que por la "orilla/borde del océano". En este sentido, según los datos del este corpus, podría establecerse una relación mucho más estrecha entre "pasear" y playa como "borde del mar" que entre "pasear" y "borde del océano"6.
Respecto del primer ejemplo presentado, en estricto rigor, los personajes descritos pasean por el mar, pero un mar "abierto y oscuro", tan hostil como la pradera africana. Existe claramente un lenguaje poético que busca aludir a los dioses mediante la denominación "señores del océano". En este sentido, océano adquiere precisamente el matiz semántico al que se hizo referencia antes y que lo hace poco compatible con "pasear": inhóspito, rudo, hostil. Véase la Figura 5:

5 En casos como el primer ejemplo citado en (2), nos inclinamos por la aparición de mecanismos de coacción. El lector interesado puede acudir a De Miguel (2009) donde se incluye una descripción de los mecanismos generativos propuestos por la TLG, con ejemplos del español.

6 De esta forma, podemos apoyar nuestras apreciaciones en la definición que el DLE ofrece de playa: "Ribera de mar (...) formada de arenales en superficie casi plana.". 
Figura 5

\section{Detalle de un ejemplo combinatorio "pasear" y océano}

Robé una mirada furtiva al mar abierto, oscuro en la noche, y me imaginé a los señores del océano paseando en la negrura como leones en la pradera africana.

Fuente: Corpus Corpes XXI (Corpes XXI, s.f.)

Hecha la aclaración, se presenta a continuación una tabla que sintetiza los resultados obtenidos al combinar mar, océano, monte y montaña con los verbos "llegar" y "pasear" (Tabla 1) 7 .

Tabla 1

Síntesis de resultados combinatorios entre verbos y sustantivos

\begin{tabular}{|l|l|l|l|l|}
\hline & monte & montaña & mar & océano \\
\hline Ilegar & 64 & 78 & 314 & 51 \\
\hline pasear & 7 & 2 & 16 & 3 \\
\hline
\end{tabular}

Fuente: Elaboración propia

A partir del ejercicio combinatorio realizado, se evidencia que, según los datos del Corpes XXI, no se suele combinar montaña y océano con el verbo "pasear". Surge, por tanto, la siguiente pregunta: ¿qué rasgo posee este verbo que le permite compatibilizar mejor con monte y mar que con montaña y océano? Para responder a esa inquietud, se podría hacer una prueba más para cada uno de los pares de palabras que se están estudiando, bajo las mismas condiciones, pero buscando posibilidades combinatorias con adjetivos que tienen un matiz de "hostilidad".

\section{montaña / monte}

Antes de comenzar con este ejercicio, resulta necesario destacar que el lema montaña posee 13312 apariciones en el Corpes XXI frente a las 6956 de monte. Debido a esto, y para evitar sesgos en la interpretación de los datos, se expresará la cantidad de coapariciones por cada mil resultados, de acuerdo con la fórmula que se presenta en la figura 6 .

\section{Figura 6 \\ Fórmula para expresar las coapariciones del sustantivo}

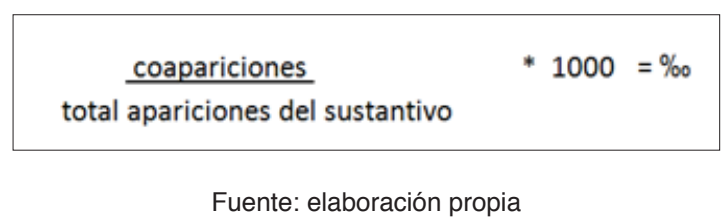

Así, según el ejemplo, las coapariciones alto-montaña se expresan de la siguiente forma (Figura 7):

Figura 7

Ejemplo de aplicación de la fórmula

\begin{tabular}{|c|c|c|}
$\frac{654}{13312}$ & $* 1000$ & \\
Fuente: elaboración propia &
\end{tabular}

Una vez presentadas las decisiones metodológicas, se ofrece una tabla que expone combinaciones con adjetivos "hostiles" para evidenciar el comportamiento de los sustantivos montaña y monte (Tabla 2).

Tabla 2

Síntesis de resultados combinatorios entre sustantivos y adjetivos

\begin{tabular}{|c|c|c|}
\hline & $\begin{array}{l}\text { montaña } 13312 \\
\text { \%o (casos) }\end{array}$ & $\begin{array}{l}\text { monte } 6956 \\
\% \text { (casos) }\end{array}$ \\
\hline alto & $49,1 \%$ (654) & $6,3 \%$ (44) \\
\hline nevado & 5,3\%о (71) & $1,3 \%$ (9) \\
\hline rocoso & 5,1\%о (68) & $0,8 \%$ (6) \\
\hline inaccesible & 0,6\%о (9) & 0\% (0) \\
\hline salvaje & 0,6\%о (8) & $0 \%$ o $(0)$ \\
\hline Inhóspito & 0,4\%о (6) & 0\% (0) \\
\hline
\end{tabular}

Fuente: elaboración propia

\section{mar / océano}

Como se mencionó antes, el lema océano tiene 4 957 entradas en el Corpes XXI, en tanto que mar sextuplica esa cantidad con 31334 resultados. En este sentido, se compararán las coapariciones entre 
los lemas en estudio y los adjetivos que se han seleccionado como representantes de la expresión del sentimiento de peligro u hostilidad, haciendo uso de la misma metodología que en el caso anterior, vale decir, expresando las coapariciones por cada mil (\%o) registros del sustantivo estudiado (Tabla 3).

Tabla 3

\section{Comparación de coapariciones océano y mar}

\begin{tabular}{|l|l|l|}
\hline & océano & Mar \\
\hline Lejano & $2,2 \%$ o & $2 \%$ o \\
\hline Profundo & $10,2 \%$ o & $3,2 \%$ o \\
\hline Oscuro & $2,6 \%$ o & $2,7 \%$ o \\
\hline Amenazante & $0,2 \%$ 0,003\% \\
\hline
\end{tabular}

Fuente: elaboración propia

Sobre la base de los datos presentados, se puede señalar que los hablantes suelen relacionar el concepto de montaña con propiedades "hostiles" con mayor facilidad que el lema monte. Tanto es así, que Corpes XXI no registra casos de combinación de monte con los adjetivos inaccesible, inhóspito o salvaje, pero sí de montaña. Por su parte, el mismo corpus demuestra que los hablantes relacionan océano con adjetivos como profundo o amenazante.

A partir de los antecedentes anteriores, se destaca -gracias a los datos que las combinaciones sintácticas ofrecen- que se puede llegar a delimitar la definición de cada una de las entradas mediante sus posibilidades combinatorias con el verbo "pasear". Para ahondar en esto, se ofrece la estructura argumental y temática de este, siguiendo el modelo expuesto en Batiukova y De Miguel (2013, p. 441) (figura 8):

\section{Figura 8}

Estructura argumental del verbo "pasear"

\begin{tabular}{|c|c|c|c|}
\hline EA: & N1 & ("pasear") & por N2 \\
\hline ET : & N1: tema & & trayecto \\
\hline
\end{tabular}

Fuente: elaboración propia
Las posibilidades combinatorias de los lemas monte-montaña y mar-océano con el verbo "pasear" se explican siguiendo el modelo expuesto por Batiukova y De Miguel (2013), que caracteriza la información subléxica que ofrece la estructura de qualia: los referentes de los cuatro nombres cuya coaparición con "pasear" se están analizando, representan un objeto natural (quale agentivo) en un caso constituyen una gran elevación (quale formal) de terreno (quale constitutivo) y en el otro una gran extensión (quale formal) de agua (quale constitutivo). Sin embargo, la baja frecuencia de aparición entre "pasear" y montaña se justifica por diferencias en el quale formal, pues montaña y océano se vinculan por los hablantes con adjetivos como alto, rocoso / profundo, amenazante... con mayor facilidad que monte y mar con esos mismos adjetivos. Se desprende de lo anterior que montaña y océano son sustantivos con una constitución más inhóspita y hostil que la de monte y mar. En consecuencia, "pasear", dado el matiz recreativo que posee, no se relaciona fácilmente con montaña u océano, pues se combina con sustantivos que contengan en su estructura temática un N2 que cumpla no solo con la condición de "trayecto", sino también con "favorecer la distracción, placer o ejercicio" (Figura 9).

\section{Figura 9}

\section{Ejemplo "pasear" con sustantivos [+recreativo] y [-recreativo]}

Juan pasea por el parque / \# Juan pasea por el barranco

Fuente: elaboración propia

\section{PROPUESTA DE DEFINICIÓN DEL VERBO “PASEAR"}

Hasta aquí, resulta evidente que se ha defendido que el contenido referido al matiz recreativo forma parte de la estructura de qualia del verbo "pasear", pues es este aspecto el que posibilita -o no- sus combinaciones con los sustantivos antes señalados; de hecho, ese contenido resulta fundamental al momento de ofrecer una definición, porque es el elemento diferenciador respecto de otros verbos de movimiento como, por ejemplo, "andar" ${ }^{\text {. }}$. Así, existe

$8 \quad$ Para una propuesta de definición mínima de este verbo, puede revisar UPSTAIRS (s.f.). 
un desplazamiento de un sujeto (animal o cosa) con una finalidad recreativa, independientemente de cómo se desplaza. Se observa entonces, que son solo dos los elementos indispensables: el desplazamiento y la recreación. De lo anterior, se desprende que en este caso, es posible inclinarse por ofrecer una definición mínima que rescate los elementos fundamentales que debe incluir la entrada del verbo "pasear". Desde esta perspectiva, el hablante, gracias a su competencia lingüística, será capaz de obtener todo el resto de significados que algunas obras suelen incorporar como acepciones distintas 9 : "pasearse la yegua", "pasear al niño", "pasear el andén", etcétera. A partir de lo anterior, se define "pasear" como se observa en la Figura 10:

\section{Figura 10}

\section{Definición del verbo "pasear"}

\section{Pasear}

desplazarse/moverse a pie/en vehículo/en un animal con una finalidad recreativa.

Ejemplo: Se paseó en caballo por la playa.

Fuente: elaboración propia

Como se anticipó en el apartado §3.2., la estructura argumental correspondiente es la recogida en la Figura 11:

Figura 11 Estructura argumental del verbo "pasear"

\begin{tabular}{|c|c|c|c|}
\hline EA: & N1 & (pasear) & por N2 \\
\hline ET: & N1: tema & & trayecto \\
\hline
\end{tabular}

Fuente: elaboración propia

Finalmente, gracias al ejemplo, se destaca que uno de los principales aspectos observado en estructuras gramaticales cuyo núcleo está formado por el verbo "pasear": el anteriormente denominado "matiz recreativo" condiciona la selección léxica que conforman los complementos que acompañan al verbo.

\section{IMPLICACIONES LEXICOGRÁFICAS Y LEXICOLÓGICAS}

Este trabajo se ha desarrollado en dos grandes áreas: por un lado, la posibilidad de establecer límites en el momento de definir sustantivos de difícil delimitación como montaña-monte y mar-océano utilizando criterios lingüísticos fundamentados en la TLG; por otro, la manera en que el estudio del verbo "pasear" presenta soluciones a esto, pues se analiza como una herramienta que, mediante las posibilidades combinatorias, permite identificar la presencia del rasgo [+ recreativo] en el nombre que materializa el argumento trayecto del verbo, y contribuir con ello a elaborar definiciones lexicográficas más precisas y con fundamento en la lingüística.

Sumado a lo anterior, desde una perspectiva lexicológica, este trabajo permite reflexionar en torno a la capacidad determinativa que adquiere el verbo "pasear" cuando funciona como núcleo de una oración. Así, la revisión de los ejemplos tomados de los corpus permite deducir que las unidades léxicas que acompañan al verbo en cuestión suelen compartir el rasgo denominado [+ recreativo] y, cuando eso no se cumple, se debe a algún interés particular (ironizar, por ejemplo) o se recurre a distintos mecanismos lingüísticos con los cuales se suple esa incongruencia. Para ilustrar estas ideas, basta con que retomar el ejemplo propuesto en la formulación de nuestra definición de "pasear" algunas líneas atrás: "se paseó en caballo por la playa".

Al repasar el caso expuesto, se observaque tanto caballo como playa poseen el rasgo [+ recreativo]: evidentemente, un caballo es un animal domesticable y usualmente utilizado para "pasear"; una playa, un espacio ameno, agradable y asociado al descanso. "Pasear en caballo por la playa" constituye, pues, una coaparición correcta, en la que se respeta la concordancia entre los rasgos léxicos de las palabras.

Siguiendo este camino reflexivo, podría explicarse la escasa compatibilidad entre "pasear" y océano, y su consiguiente casi nula presencia en los corpus de ambos lemas en una misma oración. Efectivamente, son muy pocos los ejemplos en el Corpes XXI de oraciones que posean ambos lemas como parte de 
sus elementos constituyentes, tal y como refleja la búsqueda de casos en que exista un intervalo de proximidad de 10 o menos palabras a la izquierda o derecha entre ambos lemas ${ }^{10}$. Los resultados se presentan en las figuras 12 y 13 :

Figura 12

Coapariciones "pasear" y océano

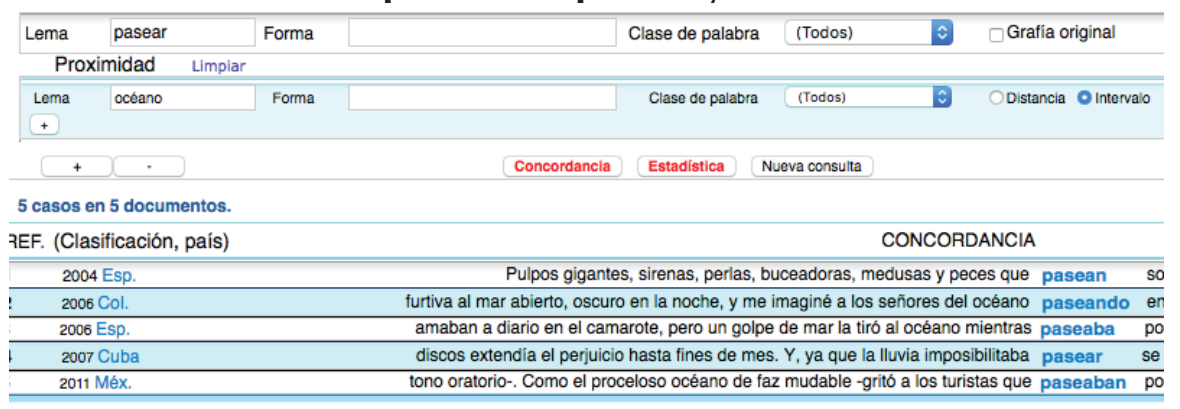

Fuente: Corpus Corpes XXI (Corpes XXI, s.f.)

Figura 13

Coapariciones "pasear" y mar

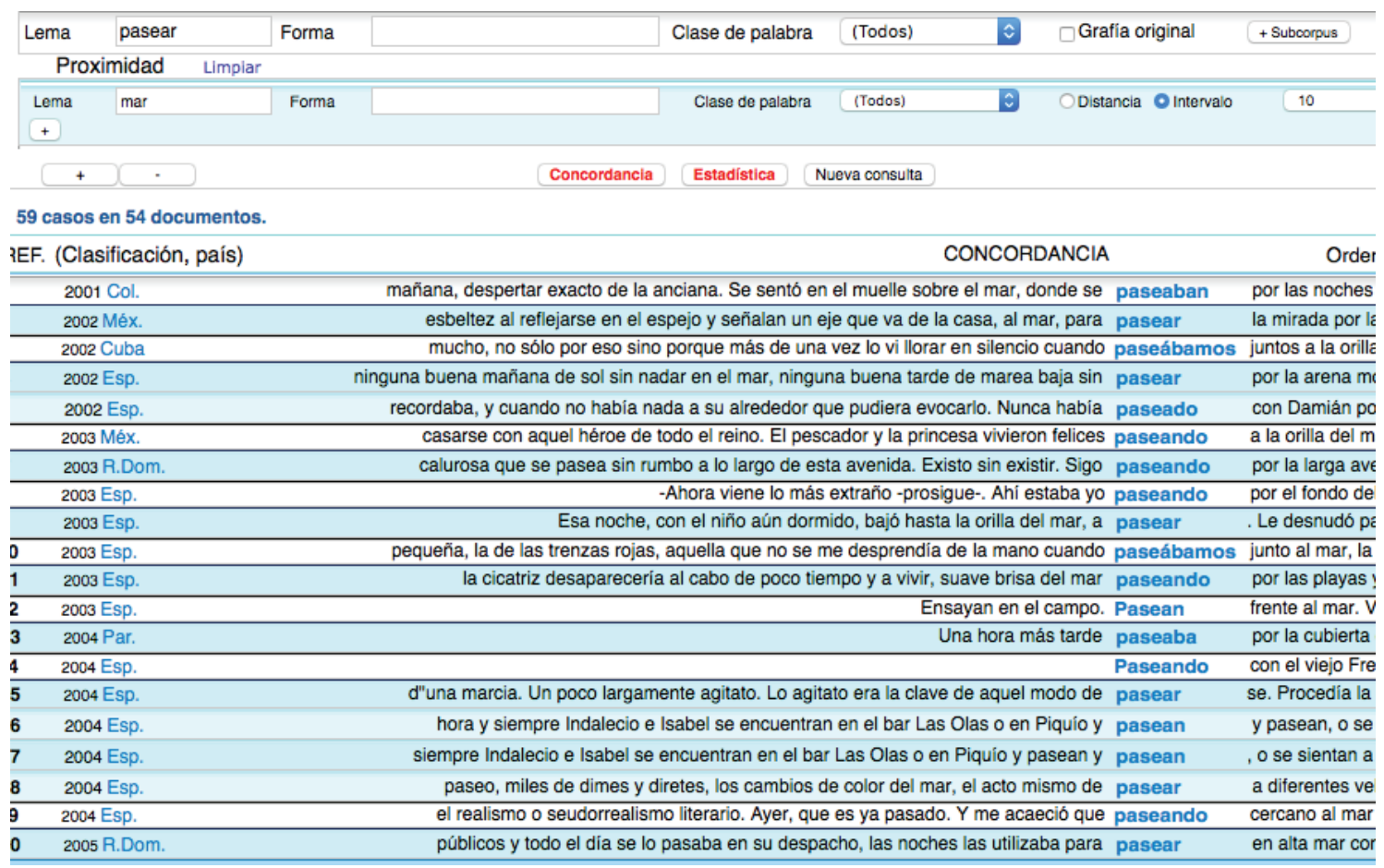

Fuente: Corpus Corpes XXI (Corpes XXI, s.f.)

Lo que resulta muy interesante de la búsqueda realizada es no solo que son muy pocos los casos de coaparición de "pasear" y océano (5 registros), sino que son muy pocos en comparación con los casos de "pasear" y mar (59 registros).
Este dato es especialmente Ilamativo porque, en principio, el significado de "pasear" debería ser también incompatible con el de mar, al menos de acuerdo con las definiciones lexicográficas habituales, que consideran "pasear" como hipónimo de

10 Un rango de \pm 10 palabras a izquierda y derecha es la amplitud máxima de búsqueda por proximidad que soporta Corpes XXI. 
"andar": en efecto, por el mar no se anda, se "nada" o "navega". El hecho de que sí se documenten en los corpus combinaciones de "pasear" y mar implica que el significado mínimo del verbo es el de desplazarse (con independencia del modo) y el de recreación, que se considera compatible con mar como argumento-trayecto.

En suma, el hecho de que mar y "pasear" sean compatibles es tanto o más importante a efectos de establecer la definición mínima del verbo que el hecho de que mar y océano no sean compatibles: uno y otro comportamiento confirman que lo que determina la compatibilidad entre verbo y nombre es la presencia o ausencia del rasgo de recreación y no del medio (acuático/terrestre), que carece de influencia para determinar la legitimidad de la coaparición. Es el mismo rasgo que determina la compatibilidad entre monte y "pasear" frente a montaña y "pasear", lo que lo hace además regular y productivo.

Desde esta perspectiva, "pasear" es hipónimo de "desplazarse" y no de "nadar", lo que debería reflejarse en las definiciones lexicográficas y, desde luego, en la propuesta de definición de este trabajo, que se recogió supra §4; a continuación, se propone una nueva definición mínima de "pasear", aún menos especificada que la anterior $y$, por consiguiente, más elástica y permeable a la combinación y generación de nuevos sentidos figurados o metafóricos, la que se elaboró siguiendo los lineamientos de la TLG y tomando como referente el Diccionario Dicemto (UPSTAIRS, 2013):

\section{Figura 14}

\section{Definición mínima del verbo "pasear"}

$$
\begin{aligned}
& \text { "Pasear" } \\
& \text { Desplazarse por algún medio con una finalidad } \\
& \text { recreativa. } \\
& \text { Ejemplo: "Se paseó en caballo por la playa". }
\end{aligned}
$$

Fuente: Elaboración propia

Finalmente, una vez establecida la definición mínima de "pasear" y sus rasgos subléxicos determinantes, se retoman aquí dos ejemplos mencionados en el apartado §2 (Figura 15):

\section{Figura 15 Ejemplos tomados de Corpes XXI}

- La Ley 16.088 fue el fruto de mi decisión de terminar con la peligrosísima extravagancia de "pasear" leonas y hasta una pantera.

- Lo que Buxton encuentra es algo inaudito: un león "paseando" por un camino.

Fuente: Corpus Corpes XXI (Corpes XXI, s.f.)

En el primer caso, se pasean dos animales que no son "paseables", es decir, que no poseen el rasgo [+ recreativo]: un león está categorizado como animal salvaje y no como doméstico, y parece incompatible con el evento denotado por "pasearlo" (en este caso, en su uso transitivo). La incongruencia, en este caso, permite catalogar el paseo como una "peligrosísima extravagancia". Luego, el problema semántico que genera la oración "un león paseando por un camino" se supera gracias a lo "inaudito" (y peligroso) que resulta un animal [-recreativo] paseando [+ recreativo] por un camino [+ recreativo]. Si la coaparición entre león y "pasear" se diera en un trayecto que constituye el hábitat habitual de un animal salvaje en libertad, como "un león paseando por la sabana", no habría en ella nada de inaudito.

Sin ánimos de extender este trabajo, se cierra con algunos ejemplos de prensa cuyo valor noticioso se puede atribuir precisamente al hecho de que se vulneran algunas de las condiciones que legitiman la combinación entre "pasear" y sus argumentos (tema y trayecto); la vulneración produce un efecto informativamente relevante: así, en los dos primeros ejemplos de la figura 16 el sujeto se pasea, inesperada, inconsciente o arriesgadamente, por un espacio [- recreativo], y en el tercero de los ejemplos, es un sujeto [- recreativo] el que describe un evento inesperado o peligroso, al pasear por un espacio ajeno a su hábitat: 


\section{Figura 16 \\ Ejemplos de prensa "pasear" + argumento [-recreativo]}

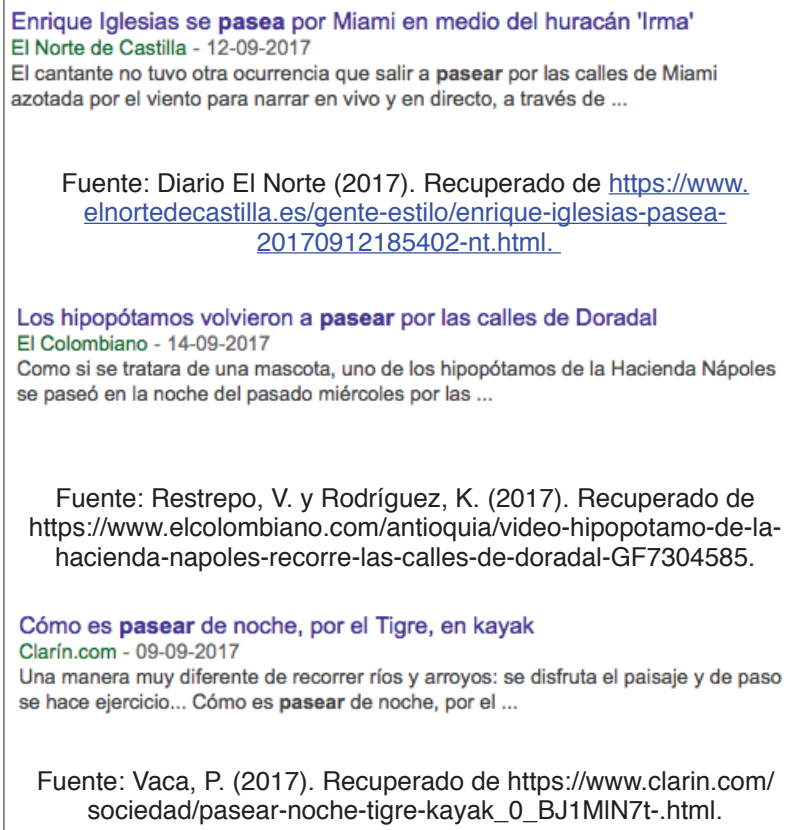

Fuente: Diario El Norte (2017). Recuperado de https://www. elnortedecastilla.es/gente-estilo/enrique-iglesias-pasea20170912185402-nt.html.

Los hipopótamos volvieron a pasear por las calles de Doradal El Colombiano - 14-09-2017

Como si se tratara de una mascota, uno de los hipopótamos de la Hacienda Nápoles se paseó en la noche del pasado miércoles por las ...

Fuente: Restrepo, V. y Rodríguez, K. (2017). Recuperado de https://www.elcolombiano.com/antioquia/video-hipopotamo-de-lahacienda-napoles-recorre-las-calles-de-doradal-GF7304585.

Cómo es pasear de noche, por el Tigre, en kayak

Clarín.com - 09-09-2017

Una manera muy diferente de recorrer ríos y arroyos: se disfruta el paisaje y de paso

se hace ejercicio... Cómo es pasear de noche, por el ...

Fuente: Vaca, P. (2017). Recuperado de https://www.clarin.com/ sociedad/pasear-noche-tigre-kayak_0_BJ1MIN7t-.html.

\section{CONCLUSIONES Y PROYECCIONES}

Luego de la revisión crítica realizada desde la TLG, se llega a la conclusión de que es importante lograr una definición mínima de los pares monte-montaña y mar-océano que recoja como parte de su estructura de qualia su origen natural (quale agentivo), su constitución interna (quale constitutivo) y sus rasgos físicos y clasificación ontológica (quale formal), pero que considere este último rasgo como el elemento que establece el límite entre cada par de lemas. Además, se prefiere una definición mínima del verbo "pasear" que tome en cuenta los dos aspectos fundamentales de su significado, vale decir, un desplazamiento cuyo objetivo es recreativo, definición mínima que nos tomamos la libertad de proponer. Todo esto, fundado en las combinaciones revisadas en el Corpes XXI, particularmente, con los verbos de movimiento "llegar" y "pasear". Asimismo, es opinión de quien escribe que la definición mínima no necesita incluir las acepciones metafóricas ni metonímicas pues, en la mayoría de los casos, resultan fácilmente deducibles por los hablantes a partir de la definición básica.
En otro ámbito, se considera que este trabajo invita a reflexionar en torno al valor que puede adquirir en los estudios lexicológicos la inclusión del rasgo [ \pm recreativo], pues podría contribuir no solo con el establecimiento de límites en entradas que poseen difusas líneas fronterizas, sino también en los estudios de semántica léxica y composicional al profundizar en el carácter determinante que le imprime el verbo "pasear" a las oraciones en las que funciona como núcleo. Una posible línea de investigación futura abierta por este trabajo podría ser, en efecto, la de indagar en la naturaleza lingüística de dicho rasgo y sus consecuencias en la legitimación de otras combinaciones, como las que ilustra los contrastes "disfrutar unas horas junto al mar" / "??disfrutar unas horas en la cárcel"; "disfrutar la conferencia en el Ateneo" / "?? disfrutar la intervención en el quirófano".

\section{REFERENCIAS BIBLIOGRÁFICAS}

Batiukova, O. y De Miguel, E. (2013). Tratamiento lexicográfico de verbos de movimiento con significado amplio. En Cabedo, A., Aguilar, M. y López-Navarro, E. (eds.), Estudios de lingüística: investigaciones, propuestas y aplicaciones (pp. 439-450). Recuperado de https://www.researchgate.net/publication/330005491_Tratamiento_lexicografico_de_verbos_de_movimiento_con_significado_amplio.

Climent, S. (1999). Marco teórico: el lexicón generativo. Recuperado de http://elies.rediris.es/elies8/ index.html.

Corpes XXI (s.f.) Corpus del Español del Siglo XXI. Visitado el 2 de mayo de 2017 en http://www.rae. es/recursos/banco-de-datos/corpes-xxi.

De Miguel, E. (2009). La teoría del lexicón generativo. En De Miguel, E. (ed.), Panorama de la lexicología (pp. 337-370). Recuperado de https://www. uam.es/gruposinv/upstairs/upstairs2/curricula/trabajos/demiguel_2009_Panorama_III_5_TLG.pdf.

De Miguel, E. (2015). Lexicología. En GutiérrezRexach, J. (dir.), Enciclopedia de lingüística hispánica (pp. 173-185). Londres / Nueva York: Routledge Publishing Co.. 
Dicemto, Diccionario electrónico de verbos de movimiento del español. Recuperado de https:// www.uam.es/gruposinv/upstairs/diccionario.html

Martí, M. (2001). Consideraciones sobre polisemia. En Martí, M., Fernández, A. y Vásquez, G. (eds.) Lexicografía computacional y semántica (pp. 64104). Barcelona: Universidad de Barcelona.

Meya, M. (1976). Modelación del campo semántico de los verbos de movimiento. Revista Española de Lingüística, (6), 1, 145-165. Recuperado de http:// www.sel.edu.es/pdf/ene-jun-76/Meya.pdf
Moliner, M. (1967). Diccionario de uso del español. Madrid: Gredos.

Pustejovsky, J. (1995). The Generative Lexicon, Cambridge, Mass.: MIT Press.

Real Academia de la Lengua Española (2014). Diccionario de la lengua española. Madrid: Espasa.

Ullman, S. (1962). Semántica. Introducción a la ciencia del significado. Madrid: Taurus.

UPSTAIRS (s.f.). Proyecto lexicográfico electrónico multilingüe de verbos de movimiento. Visitado el 3 de mayo de 2017 en https://www.uam.es/gruposinv/upstairs/v31/index.htm. 